\title{
ON THE AMPLENESS OF HOMOGENEOUS VECTOR BUNDLES
}

BY

DENNIS M. SNOW

\begin{abstract}
A formula is proved which expresses the ampleness of a homogeneous vector bundle over $G / P$ in terms of the distance of the weights of the representation of $P$ to certain dominant weights of $G$.
\end{abstract}

Classically, a line bundle $L$ over a compact complex manifold $X$ is said to be ample if the map $X \rightarrow \mathbf{P}^{N}$ associated to the sections of some power $L^{n}$ gives an imbedding of $X$. This notion has been extended to vector bundles as well [6]. In [9] Sommese generalized the notion of ampleness to the following: Let $\mathbf{E}$ be a holomorphic vector bundle over a compact complex manifold $X$ and let $\xi(\mathbf{E})$ be the tautological line bundle over $\mathbf{P}(\mathbf{E})$. Then $\mathbf{E}$ is called $k$-ample if $\xi(\mathbf{E})^{n}$ is spanned by global sections for some $n>0$ and the map $\mathbf{P}(\mathbf{E}) \rightarrow \mathbf{P}^{N}$ associated to the sections of $\xi(\mathbf{E})^{n}$ has at most $k$-dimensional fibers. This definition coincides with ampleness in the sense of Grothendieck [6] when $k=0$.

Sommese [9] proves analogues of Barth-Lefschetz type theorems for $k$-ample vector bundles, and in [10] he shows that there is a connection between $k$-ampleness and $(k+1)$-convexity of holomorphic vector bundles: If $\mathbf{E}$ is $k$-ample, then $\mathbf{E}^{*}$ is $(k+1)$-convex (in the sense of Andreotti-Grauert). For $k=0$ the converse is also true. It should be remarked that the algebro-geometric conditions for $k$-ampleness are generally easier to verify than the differential geometric conditions for $(k+1)$ convexity.

In this paper we investigate the ampleness of homogeneous vector bundles $\mathbf{E}$ over simply-connected complex projective algebraic manifolds $X$. In this case, $X$ is isomorphic to a coset space $G / P$, where $G$ is a semisimple complex Lie group and $P$ is a parabolic subgroup of $G$. Any algebraic homogeneous vector bundle over $X$ comes from a rational representation of $P$ on a vector space $E$. We show in $\$ 2$ how to calculate the ampleness of $\mathbf{E}$, which we denote by $a(\mathbf{E})$, in terms of the relative positions of the weights in $E, \Lambda(E)$, of a maximal torus $T \subseteq P$ in the lattice of weights $\Lambda$ of $G$. One need only compute the distance, measured by the number of Weyl chamber walls crossed in the diagram of $G$, between the weights $\Lambda(E)$ and certain dominant weights in $\Lambda$.

In $\$ 3$ we review the results of Goldstein [5]. His calculation of the ampleness of the tangent bundle of $X=G / P$ was one of the main inspirations for the generalization to arbitrary homogeneous vector bundles in this paper. In $\S 4$ we specialize the

Received by the editors March 28, 1984 and, in revised form, April 25, 1985.

1980 Mathematics Subject Classification. Primary 32M10, 32L15; Secondary 14M17.

C1986 American Mathematical Society $0002-9947 / 86 \$ 1.00+\$ .25$ per page 
results of $\$ 2$ to irreducible homogeneous vector bundles. In this case the computation of $a(\mathbf{E})$ is extremely easy to carry out: If $\mu$ (respectively $\lambda$ ) is the highest weight in $E$ (respectively in $E^{*}$ ) and $\lambda$ is dominant, then

$$
a(\mathbf{E})=\operatorname{dim} X-\operatorname{index} \mu,
$$

where the index of $\mu$ is the number of positive roots $\alpha>0$ such that $(\alpha, \mu)<0$. We also show that $\mathbf{E}$ is 0 -ample when the weight $\lambda$ is regular and dominant. We conclude the paper with the classical example of Borel-Weil [8], calculating the ampleness of line bundles over $X=G / P$.

\section{Definitions and notation.}

1.1 The ampleness map. Given a finite-dimensional vector space $E$ over $\mathbf{C}$, we define the projectivization of $E$, denoted $\mathbf{P}(E)$ to be the quotient of $E^{*} \backslash 0$ by the usual action of $\mathbf{C}^{*}$. We denote the image of $z \in E^{*} \backslash 0$ in $\mathbf{P}(E)$ by $[z]$. We use the same notation for the projectivization of a vector bundle $\mathbf{E}$ over a complex manifold $X$ with fiber $E$. Let $\pi: \mathbf{P}(\mathbf{E}) \rightarrow X$ be the bundle projection (with fiber $\mathbf{P}(E)$ ) and define $\xi(\mathbf{E})^{*}$ to be the rank 1 tautological subbundle of $\pi^{*} \mathbf{E}^{*}$. Recall that if $X$ is compact, the isomorphism of vector spaces $S^{n}(E) \cong \Gamma(\mathbf{P}(E), \mathcal{O}(n))$ induces an isomorphism [6]

$$
\Gamma\left(X, S^{n}(\mathbf{E})\right) \cong \Gamma\left(\mathbf{P}(\mathbf{E}), \xi(\mathbf{E})^{n}\right), \quad n \geqslant 1 .
$$

(Here, $S^{n}(\cdot)$ denotes the $n$th symmetric power.) We denote both of these vector spaces by $V_{n}$.

Definition [9]. A holomorphic vector bundle $\mathbf{E}$ on a compact complex manifold $X$ is called $k$-ample if:

(1) $\xi(\mathbf{E})^{n}$ is spanned by global sections for some $n>0$; i.e., the evaluation map $\mathbf{P}(\mathbf{E}) \times V_{n} \rightarrow \xi(\mathbf{E})^{n}$ is surjective.

(2) The projectivization $\alpha: \mathbf{P}(\mathbf{E}) \rightarrow \mathbf{P}\left(V_{n}\right)$ of the induced map $\xi(\mathbf{E})^{-n} \rightarrow V_{n}^{*}$ has at most $k$-dimensional fibers.

The number $k$ is independent of the integer $n$ for which $\xi(\mathbf{E})^{n}$ is spanned [9]. We define the ampleness of $\mathbf{E}$, denoted $a(\mathbf{E})$, to be the minimum $k$ for which $\mathbf{E}$ is $k$-ample, and we call the map $\alpha$ the ampleness map. (If $\xi(\mathbf{E})^{n}$ is not spanned for any $n>0$, we define $a(\mathbf{E})=\operatorname{dim} X$.)

It will also be convenient for us to work with the map $\nu: \mathbf{E}^{*} \rightarrow S^{n}\left(\mathbf{E}^{*}\right) \rightarrow V_{n}^{*}$ (lifted from $\alpha$ ) defined as follows: For $z_{x} \in E_{x}^{*}, x \in X$, and $s \in V_{n}$, let $\nu\left(z_{x}\right)(s):=z_{x}^{n}\left(s_{x}\right)$. Here, $z_{x}^{n}$ stands for the image in $S^{n}\left(E^{*}\right)_{x}=S^{n}(E)_{x}^{*}$ of the $n$-fold tensor $z_{x} \otimes \cdots \otimes z_{x}$. From the definitions we easily obtain, for $z_{x} \in E_{x}^{*} \backslash 0$,

$$
\alpha\left[z_{x}\right]=\left[\nu\left(z_{x}\right)\right] \in \mathbf{P}\left(V_{n}\right), \text { and } \operatorname{dim} \alpha^{-1} \alpha\left[z_{x}\right]=\operatorname{dim} \nu^{-1} \nu\left(z_{x}\right)
$$

Thus, $\xi(\mathbf{E})^{n}$ is spanned if and only if for every $z_{x} \in E_{x}^{*} \backslash 0$ there is a section $s \in V_{n}$ such that $\nu\left(z_{x}\right)(s)=z_{x}^{n}\left(s_{x}\right) \neq 0$. Easy calculations then show that the maps $\nu$ and $\alpha$ are finite on each fiber $E_{x}^{*}$ and $\mathbf{P}(E)_{x}, x \in X$, respectively. Consequently,

$$
0 \leqslant a(\mathbf{E}) \leqslant \operatorname{dim} X \text {. }
$$

1.2 Homogeneous vector bundles. As is well known, any locally trivial fiber bundle E over a space $X$ with fiber $E$ and structure group $H$ is isomorphic (in the appropriate category) to a twisted product $P \times{ }_{H} E$, where $P$ is the associated 
principal bundle over $X$. Here the twisted product $P \times_{H} E$ is defined to be the quotient of $P \times E$ by the action $h .(p, z)=\left(p \cdot h^{-1}, h . z\right),(p, z) \in P \times E, h \in H$. We denote the image of a point $(p, z) \in P \times E$ in $P \times{ }_{H} E$ by $[p, z]$. Thus, $[p . h, z]=[p, h . z]$ for all $h \in H$. Projection onto the first factor $[p, z] \rightarrow p . H$ defines the bundle structure of $P \times{ }_{H} E$ with base $P / H=X$ and fiber $E$.

Our main interest in this paper is with homogeneous vector bundles $\mathbf{E}$ over compact projective algebraic manifolds $X$. By homogeneous we mean that the group of (algebraic) bundle automorphisms of $\mathbf{E}$ acts transitively on $X$. In this case, $X$ is isomorphic to a coset space $G / H$ where $G$ and $H$ are algebraic groups and the fiber $E$ is a rational $H$-module. Thus, $\mathbf{E}$ is isomorphic to $G \times{ }_{H} E$ as described above. The finite-dimensional vector space of sections $\Gamma(X, \mathbf{E})$ is in a canonical fashion a rational $G$-module. It is isomorphic to the induced $G$-module $\left.E\right|^{G}$ which is defined to be the vector space of all algebraic morphisms $s: G \rightarrow E$ which satisfy $s\left(g h^{-1}\right)=$ $h . s(g)$ for all $g \in G, h \in H$. The action of $G$ on $\left.E\right|^{G}$ is by $(g . s)\left(g_{0}\right):=s\left(g^{-1} g_{0}\right)$, for all $g, g_{0} \in G$. The relationship between such an $s: G \rightarrow E$ and a section $\sigma \in \Gamma(X, \mathbf{E})$ is easily seen by writing a point $x \in X$ as a coset $g H \in G / H$ and expressing $\sigma(x)=\sigma(g H)$ as an element of the twisted product $G \times{ }_{H} E: \sigma(g H)=$ $[g, s(g)]$. We shall not distinguish between these $G$-modules and write $\left.e\right|^{G}$ (or sometimes $\left.E_{H}\right|^{G}$ when we want to emphasize that $E$ is an $H$-module) for $\Gamma(X, \mathbf{E})$.

Evaluating a section $\sigma$ at the identity coset $1 H$ corresponds to the $H$-module homomorphism

$$
\varepsilon:\left.E_{H}\right|^{G} \rightarrow E ; \quad \varepsilon(s):=s(1)
$$

Indeed, we have $\varepsilon(h . s)=(h . s)(1)=s\left(h^{-1}\right)=h . s(1)=h . \varepsilon(s)$. A homogeneous vector bundle is then spanned by global sections if and only if $\varepsilon$ is surjective. The induced module has the following universal property: Given any $G$-module $M$ and any $H$-module homomorphism $\varphi: M \rightarrow E$, there exists a unique $G$-module homomorphism $\varphi^{\wedge}:\left.M \rightarrow E_{H}\right|^{G}$ such that $\varphi=\varepsilon \circ \varphi^{\wedge}$ (for $m \in M$ define $\varphi^{\wedge}(m): G \rightarrow E$ by $\left.\varphi^{\wedge}(m)(g)=\varphi\left(g^{-1} m\right)\right)$. Also, if $E \rightarrow F$ is an $H$-module homomorphism, then there is an obvious $G$-module homomorphism of the induced $G$-modules $\left.\left.E\right|^{G} \rightarrow F\right|^{G}$ which commutes with the evaluation maps (see e.g. [4]).

1.3 Semisimple Lie groups. From now on, $G$ will denote a connected, simply-connected, semisimple complex Lie group. General references for this section are $[1,7,3]$. We fix a Borel subgroup $B \subset G$ and a maximal torus $T \subset B$. Let $\Lambda$ denote the character group of $T$ which we write additively. The elements of $\Lambda$ are called weights. Let $\Phi \subset \Lambda$ denote the roots of $G$ relative to $T$, and $\Phi^{+}$(resp. $\Phi^{-}$) the positive (resp. negative) roots with respect to $B$. We write $\alpha>0$ (resp. $\alpha<0$ ) if $\alpha \in \Phi^{+}$(resp. $\alpha \in \Phi^{-}$). Further, let $\Delta=\left\{\alpha_{1}, \ldots, \alpha_{l}\right\}$ denote the subset of simple roots, $l=\operatorname{dim} T=\operatorname{rank} G$, so that each $\alpha \in \Phi$ can be expressed uniquely as an integral combination $\alpha=\sum n_{i} \alpha_{i}$ with either all $n_{i} \geqslant 0$ (i.e., $\alpha>0$ ) or all $n_{i} \leqslant 0$ (i.e., $\alpha<0)$. The $\mathbf{Z}$-module $\Lambda$ is a free abelian group of rank $l$ with a basis $\left\{\lambda_{1}, \ldots, \lambda_{l}\right\}$ consisting of fundamental dominant weights, where $2\left(\lambda_{i}, \alpha_{j}\right) /\left(\alpha_{j}, \alpha_{j}\right)=\delta_{i j}$. The lattice $\Lambda$ is partially ordered by: $\lambda>\mu$ if $\lambda-\mu$ is a sum of positive roots. A weight $\sum n_{i} \lambda_{i}$ is said to be dominant if all $n_{i} \geqslant 0$. We denote the dominant weights by $\Lambda^{+}$. 
The Weyl group $W:=N_{G}(T) / T$ of $G$ acts on $\Lambda$ and can be realized as the finite linear group generated by the reflections $\sigma_{\alpha}$ of $F:=\mathbf{R} \otimes_{\mathbf{z}} \Lambda$ through $\alpha \in \Phi$. For each $\omega \in W$, we denote by $\eta_{\omega} \in N(T)$ a fixed representative of $\omega$. We let $\sigma_{1}, \ldots, \sigma_{l}$ denote the simple reflections, i.e., the reflections of $F$ through the simple roots $\alpha_{1}, \ldots, \alpha_{l}$, respectively. Every $\omega \in W$ can thus be written as a product of simple reflections $\omega=\sigma_{i(1)} \cdots \sigma_{i(j)}$, and the smallest number $j$ is called the length of $\omega$, denoted $l(\omega)$. Let $B=T \ltimes U$ be the semidirect product decomposition of $B$ where $U$ is the unipotent radical of $B$. ( $U$ is just the product of all the root groups $U_{\alpha}$, $\alpha>0$.) Then the length function satisfies

$$
\operatorname{dim} U \eta_{\omega} B=\operatorname{dim} B+l(\omega),
$$

since $l(\omega)$ is the number of positive roots which are transformed to negative roots by $\omega[3]$.

The Weyl chambers of $F$ are the closures of the connected components of $F \backslash \cup H_{\alpha}\left(\alpha \in \Phi^{+}\right)$, where $H_{\alpha}$ is the hyperplane of $F$ orthogonal to $\alpha$. The Weyl group $W$ acts simply transitively on the Weyl chambers. There is precisely one chamber, call it $F^{+}$, such that $\Lambda \cap F^{+}=\Lambda^{+}$. Thus, the $W$-orbit of any weight contains exactly one dominant weight. For any weight $\lambda \in \Lambda$ we define the index of $\lambda$ to be the number of positive roots $\alpha>0$ such that $(\alpha, \lambda)<0$ [2]. Geometrically, the index of $\lambda$ is just the number of hyperplanes $H_{\alpha}, \alpha>0$, crossed by a straight line connecting $\alpha$ to a general point of $F^{+}$. We also have the following formula:

$$
\text { index } \lambda=\min \left\{l(\omega) \mid \omega \in W, \omega . \lambda \in \Lambda^{+}\right\} .
$$

This follows from the observation that the shortest way for $\lambda$ to be moved to $\Lambda^{+}$by $W$ is by reflecting through the $s$ hyperplanes between it and $\Lambda^{+}, s=$ index $\lambda$. Now the product of these $s$ reflections can be rewritten as the product of $s$ simple reflections (see e.g. [2, p. 236]).

If $V$ is a rational $T$-module, we denote by $\Lambda(V)$ the weights of $T$ in $V$. A weight space $V_{\lambda}, \lambda \in \Lambda(V)$, is defined to be the subspace of all $v \in V$ such that $t . v=\lambda(t) v$ for all $t \in T$. The multiplicity of $V_{\lambda}$ is defined to be $\operatorname{dim} V_{\lambda}$. We often let $L_{\lambda}$ denote a $T$-stable line in $V$ of weight $\lambda$. Notice that if $V$ is a $G$-module, then $W$ permutes the elements of $\Lambda(V): \eta_{\omega} . V_{\lambda}=V_{\omega(\lambda)}$ for all $\omega \in W$. A maximal vector is a weight vector, $v \in V_{\lambda}$, which is fixed by $U, v \in V^{U}$. Let $L_{\lambda}$ be the $B$-stable line.spanned by such a $v$. Then the highest weight of the $G$-submodule $V^{\prime}$ generated by $v$ is $\lambda$, all other weights being $<\lambda$, and $L_{\lambda}$ is the unique $B$-stable line in $V^{\prime}$. Moreover, $\lambda$ is necessarily dominant with multiplicity 1 in $V^{\prime}$. The submodule $V^{\prime}$ is irreducible and isomorphic to the induced $G$-module $\left.\omega_{0} \lambda\right|^{G}$ where $\omega_{0}$ is the unique element of $W$ such that $\omega_{0}\left(\Phi^{+}\right)=\Phi^{-}$. (We are employing the convention that a weight $\mu \in \Lambda$ will also represent the (abstract) 1-dimensional $B$-module with weight $\mu$.) Note that $\omega_{0} \lambda$ is the lowest weight of $V^{\prime}$ in the sense that $\omega_{0} \lambda \leqslant \mu$ for any $\mu \in \Lambda\left(V^{\prime}\right)$.

A parabolic subgroup $G$ is a subgroup $P$ which contains a Borel subgroup. A standard parabolic subgroup $P_{I}$ is a parabolic subgroup constructed from a subset $I \subset \Delta$ of simple roots: $P_{I}$ is the subgroup generated by the fixed Borel subgroup $B$ and all the root groups $U_{\alpha}$ where $\alpha$ is a root in the $\mathbf{Z}$-linear span of $I$. Any parabolic subgroup $P$ of $G$ is conjugate to a standard parabolic subgroup $P_{I}$. Throughout this 
paper, when we refer to a parabolic subgroup of $G$ we shall assume that it is a standard parabolic subgroup. The subgroup of $W$ generated by the simple reflections $\sigma_{\alpha}$, $\alpha \in I$, will be denoted by $W_{I}$. Then $P$ is the product of its radical and a semisimple subgroup $S$, and $W_{I}$ is isomorphic to the Weyl group of $S$.

\section{The ampleness formula.}

2.1 We retain the notation of $\$ 1$. In particular, $G$ is a connected, simply-connected, complex semisimple Lie group; $B$ a Borel subgroup; $U$ the unipotent radical of $B ; P \supset B$ a parabolic subgroup; $W$ the Weyl group of $G$ relative to a fixed maximal torus $T \subset B ; l(\omega)$ the length of an element $\omega \in W ; \Lambda$ (resp. $\Lambda^{+}$) the weights (resp. the dominant weights) of $G$ relative to $T ; \Lambda(V)$ the weights of a $T$-module $V$.

In this section we derive a formula for the ampleness of a homogeneous vector bundle $\mathbf{E}=G \times{ }_{P} E$ over $G / P$ based only on the relative positions of the weights of the $P$-module $E$ in $\Lambda$. We let $\mathbf{E}_{B}:=G \times{ }_{B} E$ denote the pull-back of $\mathbf{E}$ to $G / B$. Note that the fiber of $\mathbf{E}_{B} \rightarrow \mathbf{E}$ is $P / B$ and that $\Gamma\left(G / B, \mathbf{E}_{B}\right) \cong \Gamma(G / P, \mathbf{E})$.

TheOREM. Assume $\xi(\mathbf{E})^{n}$ is spanned by global sections for some $n>0$ and let $S=\left(E^{*}\right)^{U}$. Then

(1) $\Lambda(S) \subset \Lambda^{+}$;

(2) $a\left(\mathbf{E}_{B}\right)=\max \left\{l(\omega) \mid \omega \in W\right.$ and $\omega(\mu) \in \Lambda\left(E^{*}\right)$ for some $\left.\mu \in \Lambda(S)\right\}$;

(3) $a(\mathbf{E})=a\left(\mathbf{E}_{B}\right)-\operatorname{dim} P / B$.

This result can be formulated more cleanly if we use the following notation: for any two subsets $A, B \subset \Lambda$ we will define the orbital distance between $A$ and $B$ to be

$$
d(A, B)=\min \{l(\omega) \mid \omega \in W, \omega(A) \cap B \neq \varnothing\} .
$$

Let $\omega_{0} \in W$ be the unique element such that $\omega_{0}\left(\Phi^{+}\right)=\Phi^{-}$and define $\sigma_{0}:=-\omega_{0}$, an involution on $\Lambda^{+}$.

Corollary. $a(\mathbf{E})=\operatorname{dim} G / P-d\left(\sigma_{0} \Lambda(S), \Lambda(E)\right)$.

Proof. From the Theorem,

$$
\operatorname{dim} G / P-a(\mathbf{E})=\operatorname{dim} G / B-a\left(\mathbf{E}_{B}\right),
$$

where $a(\mathbf{E})=\max \left\{l(\omega) \mid \omega \in W\right.$, and $\omega(\mu) \in \Lambda\left(E^{*}\right)$ for some $\left.\mu \in \Lambda(S)\right\}$. Now, $l\left(\omega \omega_{0}\right)=\operatorname{dim} G / B-l(\omega)[3]$, so

$$
-\max \{l(\omega)\}=\min \{-l(\omega)\}=\min \left\{l\left(\omega \omega_{0}\right)\right\}-\operatorname{dim} G / B .
$$

The formula then follows by observing that if $\tau=\omega \omega_{0}$ and $\mu \in \Lambda(S)$, then

$$
\omega(\mu)=\tau \omega_{0}(\mu) \in \Lambda\left(E^{*}\right) \Leftrightarrow \tau \sigma_{0}(\mu) \in \Lambda(E) .
$$

Thus, to compute the ampleness of a homogeneous vector bundle $\mathbf{E}=G \times{ }_{P} E$ (for which $\xi(\mathbf{E})^{n}$ is spanned), one need only find the smallest distance from the weights in $\sigma_{0} \Lambda(S) \subset \Lambda^{+}$to those in the same $W$-orbit in $\Lambda(E)$ (measured by the number of hyperplanes $H_{\alpha}, \alpha>0$, between them; see $\S 1.3$ ) and subtract this from the dimension of the base space. 
2.2 The proof of the Theorem is in two steps. The first is to examine the map $\nu$ : $\mathbf{E}^{*} \rightarrow V_{n}^{*}$ lifted from the ampleness map (§1) to obtain a formula for $a(\mathbf{E})$ in terms of $T$-stable lines in $\nu\left(E^{*}\right)$. (Here, we are identifying $E^{*}$ with $\left\{[1, z] \mid z \in E^{*}\right\} \subset G$ $\times_{P} E^{*}=\mathbf{E}^{*}$.) The second step is to translate this information into statements about the weights of $E^{*}$. The first step is contained in the following lemma. We let $L_{\mu}$ denote a $T$-stable line of weight $\mu$, and for each $\omega \in W$ we fix a representative $\eta_{\omega} \in N(T)$.

LEMMA. Let $M$ be the maximum of $l(\omega)$ for all $\omega \in W$ which satisfy $\eta_{\omega} L_{\mu} \subset \nu\left(E^{*}\right)$ for some $L_{\mu} \subset \nu\left(E^{*}\right) \cap\left(V_{n}^{*}\right)^{U}$. Then

$$
a(\mathbf{E})=M+\operatorname{dim} B-\operatorname{dim} P .
$$

Proof. Since $\alpha$ is $G$-equivariant, the fiber dimension of $\alpha$ is constant on $G$-orbits. Moreover, the fiber dimension is upper semicontinuous, so its maximum can be found by specializing within a $G$-orbit to a point on a closed $G$-orbit in $\mathbf{P}\left(V_{n}\right)$, say $G$. $[y]$ where $[y]=\alpha([z]) \in \alpha(\mathbf{P}(E))$. Since $\alpha(\mathbf{P}(E)) \cap G .[y]$ is closed and $B$-invariant, the $B$-orbits of minimal dimension in $\alpha(\mathbf{P}(E)) \cap G .[y]$ are closed. However, any closed $B$-orbit in projective space must be a $B$-fixed point. Therefore, we may assume that $y$ lies in a $B$-stable line $L_{\mu} \subset V_{n}^{*} \cap \nu\left(E^{*}\right)$ for some $\mu \in \Lambda\left(\left(V_{n}^{*}\right)^{U}\right)$. Then

$$
\begin{aligned}
\operatorname{dim} \alpha^{-1}[y] & =\operatorname{dim} \nu^{-1}(y)=\operatorname{dim}\{[g, z] \mid \nu([g, z])=y\} \\
& =\operatorname{dim}\left\{(g, z) \mid \nu(z)=g^{-1} \cdot y\right\}-\operatorname{dim} P \\
& =\operatorname{dim}\left\{g \in G \mid g \cdot y \in \nu\left(E^{*}\right)\right\}-\operatorname{dim} P .
\end{aligned}
$$

Since $\nu\left(E^{*}\right)$ is a cone, we must find the dimension of $\left\{g \in G \mid g L_{\mu} \subset \nu\left(E^{*}\right)\right\}$. By the Bruhat decomposition, each $g \in G$ lies in some $U \eta_{\omega} B, \omega \in W$, with $\operatorname{dim} U \eta_{\omega} B=$ $\operatorname{dim} B+l(\omega)(\S 1.3)$. Now,

Therefore,

$$
g L_{\mu}=u \eta_{\omega} b L_{\mu} \subset \nu\left(E^{*}\right) \Leftrightarrow \eta_{\omega} L_{\mu} \subset \nu\left(E^{*}\right) .
$$

$$
\begin{aligned}
\operatorname{dim} \nu^{-1}(y) & =\max \left\{\operatorname{dim} U \eta_{\omega} B \mid \omega \in W, \text { and } \eta_{\omega} L_{\mu} \subset \nu\left(E^{*}\right)\right\}-\operatorname{dim} P \\
& =\max \left\{l(\omega) \mid \omega \in W \text {, and } \eta_{\omega} L_{\mu} \subset \nu\left(E^{*}\right)\right\}+\operatorname{dim} B-\operatorname{dim} P .
\end{aligned}
$$

The maximum fiber dimension is then found by maximizing this number over $L_{\mu} \subset \nu\left(E^{*}\right) \cap\left(V_{n}^{*}\right)^{U}$.

The remainder of the proof of the Theorem now consists of showing that:

(a) $\Lambda(S) \subset \Lambda^{+}$; and

(b) there exists a $B$-stable line $L_{\mu} \subset \nu\left(E^{*}\right) \cap\left(V_{n}^{*}\right)^{U}$ with $\eta_{\omega} L_{\mu} \subset \nu\left(E^{*}\right)(\omega \in W)$ if and only if $\mu=n \lambda$ for some $\lambda \in \Lambda(S)$ with $\omega(\lambda) \in \Lambda\left(E^{*}\right)$.

(a) Let $\lambda \in \Lambda(S)$ and consider the surjective $B$-module homomorphism $S^{n}(E) \rightarrow$ $-n \lambda$. We then obtain a $G$-module homomorphism of induced $G$-modules $\beta: V_{n}=$ $\left.S^{n}(E)\right|^{G} \rightarrow-\left.n \lambda\right|^{G}$. Now, $\beta$ cannot be the zero map, since $\nu \mid L_{\lambda} \rightarrow \nu\left(L_{\lambda}\right)$ is finite, taking the $B$-stable line $L_{\lambda} \subset E^{*}$ to a $B$-stable line of weight $n \lambda$ in $\varepsilon_{n}^{*}\left(S^{n}(E)^{*}\right) \subset$ $V_{n}^{*}\left(\varepsilon_{n}: V_{n} \rightarrow S^{n}(E)\right.$ is the evaluation map). Furthermore, $-\left.n \lambda\right|^{G}$ is either 0 or irreducible, the latter occurring precisely when $\omega_{0}(-n \lambda)$ is a dominant weight, i.e., when $\lambda \in \Lambda^{+}$. This proves (a). 
(b) Sufficiency $(\Leftarrow)$ is obvious so we only prove Necessity $(\Rightarrow)$. Let $\nu_{0}:=\nu \mid E^{*}$. Then $\nu_{0}: E^{*} \rightarrow V_{n}$ is finite, $P$-equivariant, and satisfies $\nu_{0}(a z)=a^{n} \nu_{0}(z)$ for all $a \in \mathbf{C}, z \in E^{*}$ (\$1.1). Therefore, the preimage $\nu_{0}^{-1} L_{\gamma}$ of any $B$-stable line $L_{\gamma} \subset$ $\nu\left(E^{*}\right) \cap\left(V_{n}^{*}\right)^{U}$ is a 1-dimensional cone in $\left(E^{*}\right)^{U}=S$; hence each irreducible component of $\nu_{0}^{-1} L_{\gamma}$ is a $B$-stable line in $S$. Thus, if $L_{\mu}$ is a $B$-stable line in $\nu\left(E^{*}\right) \cap\left(V_{n}^{*}\right)^{U}$ with $\eta_{\omega} L_{\mu} \subset \nu\left(E^{*}\right)$ for some $\omega=W$, there is a $B$-stable line $L_{\lambda} \subset S, \lambda \in \Lambda(S)$, such that for all $t \in T, z \in L_{u}$ :

$$
\mu(t) \nu(z)=t . \nu(z)=\nu(t . z)=\nu(\lambda(t) z)=\lambda(t)^{n} \nu(z) .
$$

Therefore, $\mu=n \lambda$ (additive notation). Now, $\eta_{\omega} L_{\mu}$ has weight $\omega(\mu)=n \omega(\lambda)$, so we write $\eta_{\omega} L_{\mu}=L_{n \omega(\lambda)}$. As above, each irreducible component of $\nu_{0}^{-1}\left(L_{n \omega(\lambda)}\right)$ is a $B$-stable line in $E^{*}$; and we have, for all $t \in T, z_{1} \in \nu_{0}^{-1}\left(L_{n \omega(\lambda)}\right)$,

$$
\nu\left(t . z_{1}\right)=t \cdot \nu\left(z_{1}\right)=(\omega \lambda(t))^{n} \nu\left(z_{1}\right)=\nu\left(\omega \lambda(t) z_{1}\right) .
$$

We may thus conclude that $\omega(\lambda) \in \Lambda\left(E^{*}\right)$. This proves (b) and finishes the proof of the Theorem.

\section{Tangent bundles.}

3.1 An important class of homogeneous vector bundles is provided by the tangent bundles of homogeneous projective manifolds $G / P$, where $G$ is a semisimple complex Lie group and $P$ is a parabolic subgroup. The ampleness of such bundles was first worked out by Goldstein [5]. We now give a summary of his results to illustrate how the ampleness formula $\$ 2$ can be applied.

Let $E$ be the vector space quotient of the Lie algebra of $G$ by the Lie algebra of $P$. Then, under the adjoint representation, $E$ is a rational $P$-module, and the tangent bundle $T(G / P)$ is isomorphic to $\mathbf{E}=G \times{ }_{P} E$. This bundle is clearly spanned by global sections. If we decompose $G$ into simple factors $G=G_{1}$ $\times \cdots \times G_{m}$, then $G / P$ is isomorphic to $G_{1} / P_{1} \times \cdots \times G_{m} / P_{m}, P_{k}$ parabolic in $G_{k}$, $k=1, \ldots, m$. The tangent bundle splits correspondingly into a direct sum of $G$-stable subbundles $\mathbf{E}=\mathbf{E}_{1} \oplus \cdots \oplus \mathbf{E}_{m}$, and $E$ splits into a direct sum of $P$-submodules $E=E_{1} \oplus \cdots \oplus E_{m}$. Now,

$$
\Lambda(E)=\Lambda\left(E_{1}\right) \cup \cdots \cup \Lambda\left(E_{m}\right) \text { and } S=\left(E^{*}\right)^{U}=\left(E_{1}^{*}\right)^{U} \oplus \cdots \oplus\left(E_{m}^{*}\right)^{U} .
$$

Inspecting the coadjoint representation we find $\Lambda\left(\left(E_{k}^{*}\right)^{U}\right)=\left\{\beta_{k}\right\}$, where $\beta_{k}$ is the highest root of $G_{k}$ (always invariant under $\left.\sigma_{0}\right), k=1, \ldots, m$. Thus, $\Lambda(S)=\sigma_{0} \Lambda(S)$ $=\left\{\beta_{1}, \ldots, \beta_{k}\right\}$. Therefore, by Corollary 2.1 ,

$$
\begin{aligned}
a(\mathbf{E}) & =\operatorname{dim} G / P-d\left(\sigma_{0} \Lambda(S), \Lambda(E)\right) \\
& =\operatorname{dim} G / P-\min \left\{d\left(\beta_{k}, \Lambda\left(E_{k}\right)\right) \mid k=1, \ldots, m\right\} .
\end{aligned}
$$

Goldstein worked out a procedure for determining $d\left(\beta_{k}, \Lambda\left(E_{k}\right)\right)$ which we shall now outline (see also [11, Theorem 4.6]). To simplify notation, we shall drop the subscript $k$ in the following. On a case by case basis, it is not difficult to compute the orbital distance of $\beta$ to the negative roots [5, p. 370]:

\begin{tabular}{|c|c|c|c|c|c|c|c|c|c|}
\hline$G$ & $A_{n}$ & $B_{n}$ & $C_{n}$ & $D_{n}$ & $E_{6}$ & $E_{7}$ & $E_{8}$ & $F_{4}$ & $G_{2}$ \\
\hline$d\left(\beta, \Phi^{-}\right)$ & $n$ & $2 n-2$ & $n$ & $2 n-3$ & 11 & 17 & 29 & 8 & 3 \\
\hline
\end{tabular}


Moreover, for any long simple root $\alpha_{i}$ one can find an $\omega \in W$ such that $d\left(\beta, \Phi^{-}\right)=$ $l(\omega)$ with $\omega(\beta)=-\alpha_{i}[5,(4.3)]$. Now, if $I$ is the subset of simple roots which defines $P$, then the weights of $E$ are the negative roots of the form

$$
-\sum n_{j} \alpha_{j}, \quad \text { where } n_{j}>0 \text { for some } \alpha_{j} \in \Delta \backslash I \text {. }
$$

Therefore, if some $\alpha_{j} \in \Delta \backslash I$ is long, then by the above remark

$$
d(\beta, \Lambda(E))=d\left(\beta, \Phi^{-}\right) .
$$

If all $\alpha_{j} \in \Delta \backslash I$ are short, then find an $\omega \in W$ such that $d\left(\beta, \Phi^{-}\right)=l(\omega)$, with $\omega(\beta)=-\alpha_{i}, \alpha_{i}$ long. Again, a case by case computation shows that for $G=G_{2}$ or $B_{n}$ we need exactly one more reflection to get $\omega(\beta)$ to involve the short root $\alpha_{j}$ (there is only one short root for $G_{2}$ and $B_{n}$ so $\Delta \backslash I=\left\{\alpha_{j}\right\}$ ). If $G=C_{n}$ or $F_{4}$, we need a minimum of $d(I)$ additional reflections to get $\omega(\beta)$ to involve some $\alpha_{j} \in \Delta \backslash I$, where

$$
\begin{aligned}
d(I)= & \text { minimum number of nodes on the Dynkin diagram } \\
& \text { from an element } \alpha \in \Delta \backslash I \text { to a long root. }
\end{aligned}
$$

Using the same definition of $d(I)$ for all the different types of simple Lie groups, we can summarize the above cases in the formula

$$
d(\beta, \Lambda(E))=d\left(\beta, \Phi^{-}\right)+d(I) .
$$

\section{Irreducible homogeneous vector bundles.}

4.1 Let $P$ be a parabolic subgroup of a semisimple complex Lie group $G$. We shall call a homogeneous vector bundle $\mathbf{E}=G \times{ }_{P} E$ irreducible if $E$ is an irreducible $P$-module. Any line bundle over $G / P$ is necessarily homogeneous and irreducible. If $\mathbf{E}$ is irreducible, there is a simple criterion for $\xi(\mathbf{E})^{n}$ to be spanned.

Proposition. Let $E$ be an irreducible P-module, and let $\lambda$ be the weight of the unique B-stable line in $E^{*}$. Then the following are equivalent:

(1) $\xi(\mathbf{E})^{n}$ is spanned by global sections for some $n \geqslant 1$;

(2) $\mathbf{E}$ is spanned by global sections;

(3) $\lambda$ is a dominant weight, $\lambda \in \Lambda^{+}$.

Proof. Recall that $(1) \Rightarrow(3)$ was already proved in Theorem 2.1 , and $(2) \Rightarrow(1)$ is immediate. Thus, it remains to show (3) $\Rightarrow(2)$. Since $E$ is irreducible, it is induced from the $B$-character $-\lambda$, i.e., $E=-\left.\lambda_{B}\right|^{P}$ (see [8]). By transitivity of induction $[4,2]$ we have

$$
\left.E_{P}\right|^{G}=\left.\left(-\left.\lambda_{B}\right|^{P}\right)\right|^{G}=-\left.\lambda_{B}\right|^{G} .
$$

Recall also that $\left.E_{P}\right|^{G}=\left.E_{B}\right|^{G}$. If $\lambda \in \Lambda_{+}$, then the $P$-module (or $B$-module) homomorphism $\varepsilon:\left.E\right|^{G} \rightarrow E$ cannot be the zero map since the $B$-module homomorphism $\left.E\right|^{G}=-\left.\lambda_{B}\right|^{G} \rightarrow-\lambda_{B}$ is surjective and factors (via $\varepsilon$ ) through the $B$-module epimorphism $E \rightarrow-\lambda_{B}$. Therefore $\varepsilon$ must be surjective.

4.2 The ampleness of irreducible homogeneous vector bundles (which are spanned) can be easily computed using the formulas of $\S 2$. 
THEOREM. Let $E$ be an irreducible P-module and let $\mu$ (resp. $\lambda)$ be the weight of the unique B-stable line in $E$ (resp. $E^{*}$ ). If $\lambda$ is dominant, $\lambda \in \Lambda^{+}$, then the ampleness of $\mathbf{E}=G \times{ }_{p} E$ is given by

$$
a(\mathbf{E})=\operatorname{dim} G / P-\operatorname{index} \mu .
$$

Proof. Let $I$ be the subset of simple roots which defines $P$ and let $W_{I}$ be the corresponding subgroup of $W$ (=Weyl group of $G$ ). Because $E$ is irreducible, $\sigma_{0} \Lambda(S)=\sigma_{0} \lambda$ and

$$
W .\left(\sigma_{0} \lambda\right) \cap \Lambda(E)=W .(-\lambda) \cap \Lambda(E)=W_{I} \cdot(-\lambda) ; \quad W_{I}(-\lambda) \cap \Lambda^{+}=\sigma_{0} \lambda .
$$

Therefore, $\omega . \sigma_{0} \lambda \in \Lambda(E)$ for $\omega=W$ if and only if $\omega^{-1} \mu^{\prime} \in \Lambda^{+}$for some $\mu^{\prime} \in$ $W_{I} \cdot(-\lambda)$. Then

$$
\begin{aligned}
d\left(\sigma_{0} \Lambda(S), \Lambda(E)\right) & =\min \left\{l(\omega) \mid \omega \in W \text { and } \omega \cdot \mu^{\prime} \in \Lambda^{+} \text {for some } \mu^{\prime} \in W_{I} \cdot(-\lambda)\right\} \\
& =\min \left\{\operatorname{index} \mu^{\prime} \mid \mu^{\prime} \in W_{I} \cdot(-\lambda)\right\} .
\end{aligned}
$$

We claim that the minimum index is attained at $\mu$ which, by Corollary 2.1 , proves the Theorem. To see this, note that $-\lambda$ is the lowest weight in $E$ and satisfies $(\alpha,-\lambda) \leqslant 0$ for all positive roots $\alpha>0$. Now, since $\mu$ is the highest weight in $E$, at least as many reflections $\sigma_{\alpha}, \alpha>0$, are needed to take $-\lambda$ to $\mu$ as to take $-\lambda$ to any other weight $\mu^{\prime} \in W_{I} .(-\lambda)$. Thus, the number of $\alpha>0$ which satisfy $(\alpha, \mu) \geqslant 0$ is at least as large as the number of $\alpha>0$ which satisfy $\left(\alpha, \mu^{\prime}\right) \geqslant 0$ for any $\mu^{\prime} \in W_{I} .(-\lambda)$. In particular,

$$
\text { index } \mu=\#\{\alpha>0 \mid(\alpha, \mu)<0\} \leqslant \#\left\{\alpha>0 \mid\left(\alpha, \mu^{\prime}\right)<0\right\}=\text { index } \mu^{\prime} .
$$

4.3 A direct application of Theorem 2.1 yields the following sufficient condition for 0-ampleness:

Theorem. Let $E$ be an irreducible P-module and let $\lambda$ be the weight of the unique $B$-stable line in $E^{*}$. If $\lambda$ is regular and dominant, i.e., $\left(\lambda, \lambda_{i}\right)>0$ for every fundamental dominant weight $\lambda_{i}$, then $\mathbf{E}=G \times{ }_{P} E$ is 0 -ample.

Proof. First we observe that the stabilizer of $\lambda$ in $W$ is trivial. Also, if $I$ is the subset of simple roots which defines $P$, then $W .(\lambda) \cap \Lambda\left(E^{*}\right)=W_{I} .(\lambda)$. Therefore, if $\omega . \lambda \in \Lambda\left(E^{*}\right)$ then $\omega . \lambda=\omega^{\prime} . \lambda$ for some $\omega^{\prime} \in W_{I}$, so that $\omega=\omega^{\prime}$. Thus,

$$
\max \left\{l(\omega) \mid \omega . \lambda \in \Lambda\left(E^{*}\right)\right\}=\max \left\{l\left(\omega^{\prime}\right) \mid \omega^{\prime} \in W_{I}\right\}=\operatorname{dim} P / B .
$$

By Theorem 2.1, we obtain $a(\mathbf{E})=0$.

This Theorem shows that we need only apply Theorem 4.2 in the case where $\lambda$ is singular.

4.4 We conclude with the relatively simple class of examples of line bundles over $G / P$. Such bundles are automatically homogeneous, and their "ampleness" was already computed in 1954 [8]. For completeness, we include this calculation here.

Given any weight $\lambda \in \Lambda$, let $I$ be the subset of simple roots $\alpha$ orthogonal to $\lambda$, $(\alpha, \lambda)=0$, and denote by $P_{\lambda}$ the standard parabolic subgroup $P_{I}(\S 1.3)$. Clearly, $P_{\lambda}$ is the largest standard parabolic subgroup of $G$ which can have $\lambda$ as a character. 
Now let $\mathbf{E}=G \times{ }_{P} E$ be a line bundle over $G / P$ and let $\lambda$ be the weight of $E$. Then by Theorem 4.1, $\mathbf{E}$ (or any power of $\mathbf{E}$ ) is spanned if and only if $-\lambda$ is dominant. Note also that $P \subset P_{\lambda}$. To compute $a(\mathbf{E})$ we must find the maximum length $l(\omega)$ of a Weyl group element $\omega \in W$ such that $\omega \cdot \lambda=\lambda$. This is clearly given by the maximum of $l(\omega)$ over $\omega=W_{I}\left(W_{I}\right.$ is the stabilizer of $\lambda$ in $\left.W\right)$. But,

$$
\max \left\{l(\omega) \mid \omega \in W_{I}\right\}=\operatorname{dim} P_{I} / B \text {. }
$$

Therefore, by Theorem 2.1,

$$
a(\mathbf{E})=\operatorname{dim} P_{\lambda} / P,
$$

and $\mathbf{E}$ is 0 -ample if and only if $P=P_{\lambda}$.

\section{REFERENCES}

1. A. Borel, Linear algebraic groups, Benjamin, New York, 1969.

2. R. Bott, Homogeneous vector bundles, Ann. of Math. (2) 66 (1957), 203-248.

3. R. Carter, Simple groups of Lie type, Wiley, New York, 1972.

4. E. Cline, B. Parshall and L. Scott, Induced modules and affine quotients, Math. Ann. 230 (1977), $1-14$.

5. N. Goldstein, Ampleness and connectedness in complex $G / P$, Trans. Amer. Math. Soc. 274 (1982), $361-373$.

6. R. Hartshorne, Ample vector bundles, Inst. Hautes Études Sci. Publ. Math. 29 (1966), 63-94.

7. J. Humphreys, Linear algebraic groups, Springer-Verlag, New York, 1975.

8. J.-P. Serre, Représentations linéaires et espaces homogènes Kählerians des groupes de Lie compacts (d'après Borel et Weil), Sém. Bourbaki, Exposé 100, May 1954, Benjamin, New York, 1956.

9. A. Sommese, Submanifolds of Abelian varieties, Math. Ann. 233 (1978), 229-256.

10. __ A convexity theorem, Proc. Sympos. Pure Math., vol. 40, Amer. Math. Soc., Providence, R.I., 1983, pp. 497-505.

11. R. Steinberg, On the desingularization of the unipotent variety, Invent. Math. 36 (1976), 209-224.

Department of Mathematics, University of Notre Dame, Notre Dame, Indiana 46556 